\title{
Actuaciones del profesorado ante el bullying en contextos con y sin Equipos de Ayuda. Estudio en España y Brasil
}

\author{
Teachers' actions against bullying in contexts with and without Help \\ Teams. A study in Spain and Brazil
}

\author{
José María Avilés Martínez ${ }^{1}$ \\ Universidad de Valladolid (España) \\ Luciene R. Paulino Tognetta ${ }^{2}$, Rafael Petta Daud ${ }^{3}$ \\ Universidad Estadual de Sao Paulo (Brasil)
}

Recibido: $14-11-19$

Aceptado: $12-06-20$

\section{Resumen}

La prevención del acoso en la escuela supone implicar a quienes conviven juntos en ella en actuaciones para erradicarlo. La posición moral del profesorado ante el fenómeno aun siendo decisiva no es suficiente ya que se le hace imprescindible una actuación coherente y una gestión de los casos apropiada, donde además se implique al alumnado como protagonista en la resolución mediante la colaboración en la mejora del clima de convivencia y en la prestación de ayuda a quienes son maltratados por sus iguales. Esta investigación compara respuestas de profesorado (721) y alumnado (1.068) de España y Brasil de centros con y sin estructuras de apoyo entre iguales (Equipos de Ayuda). El profesorado responde ante un instrumento que mide su posición moral ante el bullying y el alumnado revela las actuaciones de su profesorado ante el acoso a partir del instrumento Insebull. Se observa entre el profesorado una mayor adhesión a posiciones morales ante el bullying en contextos en que funcionan estructuras de apoyo entre iguales que en los que no tienen. El alumnado percibe una mayor intervención del profesorado sobre el acoso entre el profesorado español que entre el brasileño donde actúan otras figuras. En conclusión se hace necesario implementar entre el profesorado una mayor concienciación sobre la importancia de su papel en la prevención y resolución del bullying y desarrollar y generalizar estructuras de apoyo entre iguales como

\footnotetext{
${ }^{1}$ Doctor en Psicología. Profesor en el Departamento de Psicología de la Facultad de Educación y Trabajo Social de la Universidad de Valladolid (España). Autor para Correspondencia. E-mail: aviles@uva.es ORCID: https://orcid.org/0000-0002-9067-9963

${ }^{2}$ Doctora en Psicología. Profesora de la Universidad Estadual de Sao Paulo (Brasil). E-mail: 1rpaulino@unesp.br ORCID: https://orcid.org/0000-0003-0929-4925

${ }^{3}$ Doctorando de la Universidad Estadual de Sao Paulo (Brasil). E-mail: rafael.petta@unesp.br ORCID: https://orcid.org/0000-0001-9038-3399

(C) Los autores. Este artículo es publicado por la Revista de Investigación en Psicología de la Facultad de Psicología, Universidad Nacional Mayor de San Marcos. Este es un artículo de acceso abierto, distribuido bajo los términos de la licencia Creative Commons Atribucion - No Comercia_Compartir Igual 4.0 Internacional. (http://creativecommons.org/licenses/by-nc-sa/4.0/) que permite el uso no comercial, distribución y reproducción en cualquier medio, siempre que la obra original sea debidamente citada.
} 
los Equipos de Ayuda en los centros educativos, como medida preventiva del acoso y para la mejora del clima escolar entre estudiantes.

Palabras clave: Convivencia; Profesorado; Bullying; Sistemas de Apoyo entre Iguales; Equipos de Ayuda.

\begin{abstract}
The prevention of bullying in schools implies involving the whole school communityin actions to eradicate it. Even though the moral position of the teaching staff about bullying is decisive, it is not sufficient since coherent actions and appropriate case management is necessary. This approach must also involve the students as center pieces in the resolution through collaboration in improving the atmosphere of convivencia and in providing help to those mistreated by their peers. This research compares answers from teachers (721) and students $(1,068)$ in Spain and Brazil from centers with and without peer support structures (Help Teams). Teachers give answers to an instrument that measures their moral position facing bullying and students reveal the responses of their teachers to bullying situations based on the Insebull instrument. Teachers' answers reflect a greater adherence to moral positions when dealing with bullying in contexts with support structures between peers rather than in those without them. Students perceive a greater intervention in bullying situations by Spanish teachers than by Brazilian ones because the latter count on other sources of help. In conclusion it is necessary to implement awareness among teachers of the importance of their role in the prevention and resolution of bullying as well as developing and generalizing peer support structures such as Help Teams in schools, as an effective measure to prevent bullying and improve school atmosphere among students.
\end{abstract}

Keywords: Convivencia; Teachers; Bullying; Peer Support Systems; Help Teams.

El fenómeno del maltrato entre iguales necesita ser acometido desde todos los ámbitos de intervención de forma intencional y colectiva (Avilés, 2015). Especialmente importante es el papel que juega el profesorado en la prevención y abordaje en el aula con el grupo de iguales, trasmitiendo las actitudes y mensajes adecuados y actuando de forma inequívoca ante los casos. De esta forma, globalmente definen su posición moral sobre las situaciones. En esa tarea también deben ser capaces de sumar la colaboración y el protagonismo del alumnado como parte fundamental de la estrategia de abordaje de las situaciones, facilitando la construcción de estructuras de apoyo entre iguales como vías preferentes que permitan la implicación de los espectadores en el ejercicio de la ayuda y la confrontación al acoso.

El fomento de la convivencia ética en la escuela con el fin de erradicar el bullying pasa necesariamente, aunque no solo, por un ejercicio de concienciación de todas las personas implicadas en su ocurrencia, especialmente acerca de los roles que ocupan en él, las dinámicas de funcionamiento del acoso, el manejo de las herramientas que están disponibles para acometerlo y el fomento del compromiso para combatirlo. Sin embargo, parece necesario avanzar más y profundizar en el entrenamiento efectivo del profesorado para poner en marcha actuaciones eficaces, que junto a su alumnado permitan mantener a raya esta lacra que contamina 
las relaciones interpersonales de abuso e imposición (Cowie \&, Smith, 2002). Por otra parte, es necesario seguir implementando estructuras estables de apoyo en los centros educativos que permitan facilitar la ayuda a quienes la necesitan en momentos determinados, si puede ser desde manos cercanas y similares y con la formación suficiente para prestarla (Salmivalli, Voeten, Poskiparta, 2011). En este sentido, el recorrido pendiente es evidente y la implicación del profesorado y el alumnado en esta tarea decisivas.

\section{La gestión del profesorado de las dinámicas del bullying}

Desde las distintas administraciones educativas habitualmente se vienen implementando políticas públicas para erradicar el fenómeno del maltrato entre iguales (Olweus, 1998) con el fin de dotar al profesorado de herramientas efectivas en esa lucha. Se le suele colocar como punta de lanza que ha de liderar las iniciativas en el seno de la comunidad educativa en colaboración con las familias y con el propio alumnado. Especialmente significativas son las iniciativas llevadas a cabo y que se refieren a la normativización del fenómeno (Gómez Rivas, 2011), la sensibilización, y especialmente con la formación de los agentes educativos para el desempeño de esas tareas, explícitamente con el profesorado (Zaitegi et al, 2010).

Sin embargo, al mismo tiempo, también con frecuencia, se denotan déficits en la gestión efectiva que el profesorado hace en las dinámicas bullying en su aula. En lo que tiene que ver con su gestión emocional (Gini, Albiero, Benelli y Altoé, 2007 ) y con las destrezas concretas para manejar los casos y el saber actuar ante ellos de forma constructiva y ecológica, y en la medida de lo posible, restauradora. Como si hubiera una distancia importante entre el mensaje institucional que se transmite, lo que formativamente se recibe, el posiconamiento que cada cual posee y adopta frente al fenómeno y la aplicación que es capaz de hacer en su propia aula, frente a los ojos de su propio alumnado que evalúa la efectividad de sus acciones, poniendo así de manifiesto la necesaria consideración de todos los elementos que funcionarían como factores de éxito para sus intervenciones.

Nadie duda de la importancia que el profesorado tiene como modelo moral de conducta para su alumnado. Especialmente significativas son sus actitudes ante el bullying, especialmente para la sensación de seguridad de las personas acosadas (Newman y Murray, 2005). Es sabido que algunas evaluaciones morales que en ocasiones hace el profesorado ante situaciones de bullying revelan sus formas de desconexión moral, al culpar a la víctima de lo que le pasa o al pensar que son simples bromas de ajuste social, cuando en realidad se trata de acoso encubierto.

Por otra parte, también resultan significativas las dinámicas específicas que se generan en el aula como microsistema de relaciones, como fuerzas contrapuestas que pugnan por prevalecer, cuando se inician y pretenden establecerse las dinámicas de dominio sumisión entre quienes imponen el abuso, las personas que 
se defienden de él y aquellas que lo contemplan y lo terminan consintiendo de facto (Salmivalli, Lagerspetz, Björkqvist, Österman y Kaukiainen, 1996). Que los adultos entiendan estos procesos es básico para poder intervenir adecuadamente en ellos. Pero no parece suficiente. Además, el profesorado debe incorporar las herramientas de gestión útiles, con un entrenamiento adecuado, para que sus actuaciones sean eficaces y respondan a lo que el alumnado espera.

Por eso, también se hace necesario entender las dinámicas bullying desde las perspectivas de quienes las viven, no solo desde las víctimas, para también elegir las actuaciones adultas que de forma ponderada no sean, ni demasiado reactivas, ni contemporizadoras, y ni mucho menos, ambiguas, y por tanto, supongan un catalizador que las haga más adecuadas para una resolución justa y saludable. De ahí, la necesidad de considerar la visión que el alumnado tiene de la dirección y la efectividad de las respuestas que su profesorado da al bullying.

Sin embargo, no solo el profesorado lucha contra el acoso en la escuela. Son los propios alumnos y alumnas quienes aprenden formas de defensa y tienen posición moral frente a este hecho, trabajando para erradicarlo. Desafortunadamente, muchas veces de manera individual cuando lo sufren o lo viven cerca. En otras ocasiones, de forma organizada e intencional, como es a través de algunos de los llamados Sistemas de Apoyo entre Iguales -SAI- (Avilés, 2017).

Considerar y tener presente la forma como el alumnado entiende el acoso y cómo lo gestiona y autogestiona cuando se presenta, ayuda al profesorado y a otras personas adultas a corregir y adecuar sus actuaciones para hacerlas más efectivas, individualmente y en colaboración con ellos.

\section{La actuación del profesorado como modelo desde el que construir}

Las y los adultos se muestran frente al alumnado como referentes de seguridad y norma. El profesorado tiene a su disposición su propio posicionamiento frente al bullying y el que le marca la escuela. En este sentido, tenemos presente el resultado de una investigación (Tognetta, Avilés, Gonçalves, Andrade, Daud y Santos, 2019) que sitúa al profesorado de escuelas en diferentes posiciones frente al acoso. Reflexiona sobre los mecanismos de conexión y desconexión moral (Bandura, 1999) que el profesorado usa cuando se enfrenta al bullying.

Al profesorado de esas escuelas, en la presente investigación, se le somete a una evaluación de su alumnado para valorar si sus actuaciones contra el bullying ante ellos coinciden con las posiciones morales declaradas. Esto se hace por la necesidad de encajar el discurso teórico del profesorado frente al bullying con sus acuaciones para erradicarlo, con el fin de valorar la coherencia de sus conductas. El profesorado debe, por una parte, saber trasmitir a su alumnado el mensaje contra el bullying, y por otra, actuar contra él de la forma esperada, con 
la capacitación y el entrenamiento suficientes como para afianzar su credibilidad y profesionalidad. En esta tarea, el profesorado no va a estar solo.

La cultura de la escuela y las actuaciones desde otros sectores son importantes. La ayuda del contexto es crucial, ya que facilita al profesional de la educación dirección, mensaje dominante y estructuras y objetivos por los que trabajar. En definitiva, el profesorado que se ve impelido por la ethos de la escuela, las familias y por el alumnado, de forma recíproca, a luchar contra el abuso, tendrá menos dudas de hacerlo al tener un escenario propicio y prescriptivo: el desarrollo de un proyecto de convivencia y la puesta en marcha de estructuras de lucha contra el acoso. Esta idea ahonda en el hecho de que desde cuantos más frentes se aborde el acoso de forma coordinada, mejores resultados de control llegaremos a tener sobre él. Si se ponen en marcha al mismo tiempo todos los factores que ayudan a su erradicación se estará logrando un equilibrio complementario que hará que la respuesta sea más efectiva y compensadora: el equilibrio de la ecoconvivencia (Avilés, 2019b).

Otro factor es la formación y el entrenamiento. Es necesario reconocer también que aun habiendo voluntad de intervenir, parte del profesorado no actúa por falta de conocimientos sobre cómo tratar los casos, lo que les resta eficacia (Embrace Civility, 2016). Y la percepción de autoeficacia del profesorado ante situaciones de violencia depende de su entrenamiento para afrontarla. Es significativamente menor en quienes no lo han recibido (Sela-Shayovitz, 2009). Así, estas intervenciones mal dirigidas se revelan entonces como inocuas, llegando incluso, a potenciar las situaciones de acoso que quieren combatir. En estos casos, el alumnado evita comunicarlo al profesorado, ya que desde su perspectiva el profesorado no garantiza resolver el problema.

Finalmente, otro factor de ayuda es el papel del alumnado. Las representaciones del alumnado de las actuaciones del profesorado como justas y eficaces ante las agresiones, y de cariño y respeto frente a ellos, respectivamente les permiten, por una parte una mayor solicitud de ayuda (Aceves, 2010), y por otra, una disminución de las situaciones de victimización (Gage, Prykanowsky y Larson, 2014). Incluso, cuando se valora positiva la relación profesor/alumno en clima escolar aparecen menores índices de acoso (Debarbieux, 2010; Vinha, Nunes, Tognetta y Avilés, 2017) y menor presencia de ideas suicidas y victimización. Además, el profesorado puede ayudar a construir las redes que permitan mejorar el clima escolar y el bienestar de sus miembros. Desde hace tiempo venimos trabajando en la facilitación de Sistemas de Apoyo entre Iguales (SAIs) como estructuras internas de los grupos que den protagonismo al alumnado en la gestión de su propia convivencia (Avilés, 2017, 2019a). Una de esas estructuras son los Equipos de Ayuda (Avilés, 2012, 2017, 2019a; Avilés. Torres y Vián, 2008; Avilés, Cowie y Alonso, 2019). Los constituyen tres o cuatro alumnos del grupo, que son elegidos por sus compañeros para formar un Equipo que a lo largo del curso va a estar en 
disposición de ayudarles en las problemáticas que se les planteen. Previamente, reciben la formación necesaria para realizar esa misión. El trabajo fundamental de quienes participan en este modelo es la de prestar ayuda a quienes lo necesitan o lo solicitan, empleando un catálogo de técnicas y habilidades que permiten a los ayudados, ser capaces de encontrar un camino para resolver sus problemas (Avilés y Alonso, 2017 a,b; Avilés y Petta, 2018). En una dirección de proporcionar a sus iguales modelos de actuación moral frente a la violencia, apoyando a las víctimas y generando un clima social en el grupo contra el abuso.

\section{Objetivos}

El presente estudio tiene como objetivo evaluar las diferencias entre las actuaciones del profesorado percibidas por su alumnado ante el acoso y las posiciones morales que los docentes adoptam frente al bullying, comparando dos tipos de escuelas: unas que disponen de estructuras para el trabajo de la convivencia ética en la escuela (con Equipos de Ayuda entre el alumnado -CONEA-) y otras que no las tienen (sin Equipos de Ayuda -SINEA-). Además, esta comparación se hace en dos contextos educativos distintos (brasileño y español).

\section{Hipótesis}

Se hipotetiza que el profesorado que trabaja la convivencia ética en la escuela con su alumnado a través de estructuras de apoyo entre iguales muestra menos diferencias entre posición y actuación de aquellos que actúan en contextos sin estruturas de apoyo.

Además se prevén diferencias entre las escuelas brasileñas y españolas respecto a la actuación del profesorado respecto al bullying, dado que en Brasil no existen todavía políticas públicas sistematizadas de prevención del bullying ni de implementación de programas de conviencia en las redes escolares.

\section{Participantes}

Participan en esta investigación 721 profesores y profesoras (328 de Brasil y 393 de España) y 1068 alumnos y alumnas (438 de España y 630 de Brasil) que componen dos muestras delimitadas por el criterio de conveniencia y escogidas de modo intencional entre el profesorado y el alumnado que trabaja en escuelas que tienen proyectos de convivencia (ethos) con Equipos de Ayuda y otros cuyas instituciones no poseen ese Sistema de Apoyo entre Iguales.

En ambos países, en las escuelas con Equipos de Ayuda, el profesorado recibe formación previa en la que de manera general se aportan herramientas de resolución de conflictos, reconocimiento y abordaje de situaciones de bullying y la preparación para la selección, formación y desarrollo de los Equipos de Ayuda. Igualmente el alumnado recibe un paquete formativo (Avilés y Alonso, $2017 \mathrm{a}, \mathrm{b}$ ) 
para poder desarrollar la tarea de la ayuda a sus iguales, donde aprenden a guardar la confidencialidad, reconocer los conflictos, escuchar activamente, tomar decisiones en equipo y las fases y dinámicas más efectivas para ayudar.

El profesorado y el alumnado participante forma dos grupos, tanto en Brasil como en España, - CONEA, con Equipos de Ayuda en sus escuelas y SINEA, sin Equipos de Ayuda, conforme se muestra en las Tablas 1 y 2.

Tabla1

Distribución del profesorado por países y grupos

\begin{tabular}{lcccc}
\hline \multirow{2}{*}{ Grupos } & & \multicolumn{2}{c}{ Países } & \multirow{2}{*}{ Total } \\
\cline { 2 - 4 } & & Brasil & España & \\
\hline CONEA & $\mathrm{N}$ & 55 & 129 & 184 \\
\cline { 2 - 5 } & $\%$ & $16,76 \%$ & $32,82 \%$ & $25,52 \%$ \\
\hline SINEA & $\mathrm{N}$ & 273 & 264 & 537 \\
\cline { 2 - 5 } & $\%$ & $83,24 \%$ & $67,18 \%$ & $74,49 \%$ \\
\hline Total & $\mathrm{N}$ & 328 & 393 & 721 \\
& $\%$ & $45,49 \%$ & $54,51 \%$ & $100 \%$ \\
\hline
\end{tabular}

Tabla2

Distribución del alumnado por países y grupos

\begin{tabular}{lccc}
\hline & Total España & Total Brasil & Total \\
\hline CONEA & $243(55,02 \%)$ & $500(52,2 \%)$ & 743 \\
SINEA & & & $(69,57)$ \\
Total & $195(44,98 \%)$ & $130(47,8 \%)$ & 325 \\
& & & $(30,43)$ \\
& $438(100 \%)$ & $630(100 \%)$ & $(1068 \%)$ \\
\hline
\end{tabular}

\section{Instrumentos y procedimiento}

El primero de los instrumentos recoge la posición moral del profesorado frente al bullying, se construyó ad hoc y se adapta de otro dirigido al alumnado y que ha sido validado en otras investigaciones (Tognetta, Avilés, Rosario y Alonso, 2015). Aquí únicamente nos serviremos de sus resultados a grandes rasgos y siempre para compararlo con los datos aportados por su alumnado resultantes del segundo instrumento.

El otro, el que mide las respuestas del alumnado, recoge sus posiciones en el cuestionario Insebull (Avilés y Elices, 2007) sobre las actuaciones de su profesorado ante el bullying.

En el primer instrumento, se enfrenta al profesorado a dos relatos de bullying, uno con una víctima pasiva (HA) y otro con una víctima provocativa (HB) 
y se les pide que se adhieran a las alternativas que se les ofrecen (14 alternativas) y con las que estarían de acuerdo. Estas alternativas suponen distintas posturas ante el acoso. Sus respuestas se procesan y clasifican conforme a las categorías de desconexión moral de Bandura (1999) y las categorías de conexión moral a partir de los presupuestos de moralidad formulados desde la epistemología genética (Kohlberg, 1989, 1992; Piaget, 1932).

Después de la comprobación estadística de viabilidad de los factores establecidos, se formulan cuatro grupos (niveles progresivos) en que se ubica al profesorado conforme a la 'calidad moral' de sus respuestas:

- F1 correspondiente a categorías de desconexión moral que niegan el propio contenido moral de la situación que se analiza, culpabilizando o deshumanizando a la víctima o justificando o disfrazando lo que sucede.

- F2 representa las respuestas que revelan desconexión moral aunque sin la negación del contenido moral en juego, desplazando o difundiendo responsabilidad, o minimizando o distorsionando consecuencias para la víctima.

- F3 engloba las respuestas que poseen conexión moral, aunque por convención social.

- F4 identifica las respuestas propiamente morales por principio.

Con la definición de los cuatro fatores mencionados, el primer instrumento utilizado fue sometido al programa de análisis estadístico MPLUS 6.12 y validado para ambas muestras de esta investigación (RMSEA $=0,033, \mathrm{CFI}=0,843 \mathrm{e}$ $\mathrm{TLI}=0,827$ para la muestra brasileña y $\mathrm{RMSEA}=0,039, \mathrm{CFI}=0,794$ e $\mathrm{TLI}=0,774$ para la muestra española).

El segundo de los instrumentos, el Insebull, en el item $n^{\circ} 17$ del autoinforme recoge las respuestas del alumnado cuando ve actuar a su profesorado ante situaciones de bullying en su propia aula. Determina quiénes actúan para parar el acoso.

\section{Diseño y Análisis Estadístico}

El segundo instrumento que recoge la opinión del alumnado fue validado para la muestra española $(0,84)$ con Alpha de Crombach de consistencia interna. Los datos se analizaron con el programa estadístico SAS 9.2 y el test exacto de Fisher con niveles de significancia del 5\%. Respecto a muestra brasileña la validación de este instrumento se encuentra en proceso.

\section{RESULTADOS}

En cuanto a la comparación de la 'calidad moral' de las respuestas del profesorado ante el bullying, como se observa en las Tablas 3 y 4 , se perciben, en general, 
mayores niveles de conexión moral a favor de quienes gestionan escuelas con Equipos de Ayuda. Las diferencias son más significativas entre el profesorado de la muestra brasileña $(\mathrm{p}<0,01$ para HA y HB) que española $(\mathrm{p}=0,36$ para HA y $\mathrm{p}=0,48$ para $\mathrm{HB})$.

\section{Tabla 3}

Comparación entre profesorado brasileño y español de escuelas sin Equipos de Ayuda, conforme a las historias y a sus respetivos niveles

\begin{tabular}{cccccc}
\hline Historias & Niveles & $\begin{array}{c}\text { Brasil } \\
(\mathbf{n = 2 7 3 )}\end{array}$ & $\begin{array}{c}\text { España } \\
(\mathbf{n = 2 6 4 )}\end{array}$ & Total & Valor *p \\
\hline \multirow{2}{*}{ Historia A (HA) } & F 1 & $3(1,1 \%)$ & $2(0,76 \%)$ & $5(0,93 \%)$ & \\
& F 2 & $167(61,17 \%)$ & $62(23,48 \%)$ & $229(42,64 \%)$ & $<0,01$ \\
& F 3 & $61(22,34 \%)$ & $16(6,06 \%)$ & $77(14,34 \%)$ & \\
F 4 & $42(15,38 \%)$ & $184(69,7 \%)$ & $226(42,09 \%)$ & $<0,01$ \\
\hline
\end{tabular}

\section{Tabla 4}

Comparación entre profesorado brasileño y español de escuelas con Equipos de Ayuda, conforme a las historias y sus respectivos niveles

\begin{tabular}{cccccc}
\hline Historias & Niveles & $\begin{array}{c}\text { Brasil } \\
(\mathbf{n = 5 5})\end{array}$ & $\begin{array}{c}\text { Espana } \\
(\mathbf{n = 1 2 9 )}\end{array}$ & Total & Valor *p \\
\hline & F 1 & $1(1,82 \%)$ & $3(2,33 \%)$ & $4(2,17 \%)$ & \\
Historia A (HA) & F 2 & $20(36,36 \%)$ & $24(18,6 \%)$ & $44(23,91 \%)$ & $<0,01$ \\
& F 3 & $13(23,64 \%)$ & $10(7,75 \%)$ & $23(12,5 \%)$ & \\
& F 4 & $21(38,18 \%)$ & $92(71,32 \%)$ & $113(61,41 \%)$ & \\
Historia B (HB) & F 1 & $0(0 \%)$ & $1(0,78 \%)$ & $1(0,54 \%)$ & \\
& F 2 & $11(20 \%)$ & $21(16,28 \%)$ & $32(17,39 \%)$ & 0,68 \\
& F 3 & $5(9,09 \%)$ & $19(14,73 \%)$ & $24(13,04 \%)$ & \\
\hline
\end{tabular}

Respecto a la intervención para parar las situaciones de acoso entre iguales como se observa en la Tabla 5, los resultados muestran que en la percepción que tiene el alumnado de las actuaciones de su profesorado, se constatan diferencias significativas entre países en la inacción ante las situaciones, especialmente cuando en los centros no funciona una estructura de ayuda. Se encontraron diferencias significativas $(p<0,01)$ entre las muestras con y sin Equipos de Ayuda en cada país, así como entre las muestras brasileña y española $(\mathrm{p}<0.01)$.

Tanto si hay Equipos de Ayuda como si no, la percepción del alumnado es que se produce una marcada diferencia para parar el acoso entre el profesorado de ambos países a favor del español. En Brasil, en los centros sin estructuras, los alumnos indican que la intervención para parar el bullying corre más a cargo de otras personas que actúan en las escuelas que de su profesorado.También en Brasil, son las mujeres las que intervienen más que los hombres, sean docentes o estudiantes, en los centros donde no hay estructuras. 


\section{Tabla 5}

Respuestas en el Insebull en Brasil y en España en contextos CON y SIN Equipos de Ayuda.

\begin{tabular}{|c|c|c|c|c|c|c|c|c|}
\hline \multicolumn{9}{|c|}{ Item 17 ( ¿Quién actúa para parar las situaciones de intimidación?) } \\
\hline & \multicolumn{2}{|c|}{ BRASIL } & \multirow{2}{*}{ Total } & & \multicolumn{2}{|c|}{ ESPAÑA } & \multirow{2}{*}{ Total } & \\
\hline & SIN & $\mathrm{CON}$ & & & SIN & $\mathrm{CON}$ & & \\
\hline Nadie & $\begin{array}{c}101 \\
(24,3 \%)\end{array}$ & $\begin{array}{c}93 \\
(20,9 \%)\end{array}$ & 194 & & $\begin{array}{c}18 \\
(9,5 \%)\end{array}$ & $\begin{array}{c}53 \\
(22,4 \%)\end{array}$ & 71 & \\
\hline Algún profesor & $\begin{array}{c}22 \\
(5,3 \%)\end{array}$ & $\begin{array}{c}26 \\
(5,8 \%)\end{array}$ & 48 & & $\begin{array}{c}33 \\
(17,4 \%)\end{array}$ & $\begin{array}{c}60 \\
(25,3 \%)\end{array}$ & 93 & \\
\hline Alguna profesora & $\begin{array}{c}28 \\
(6,7 \%)\end{array}$ & $\begin{array}{c}11 \\
(2,5 \%)\end{array}$ & 39 & $\stackrel{\overrightarrow{0}}{\dot{\theta}}$ & $\begin{array}{c}14 \\
(7,4 \%)\end{array}$ & $\begin{array}{c}5 \\
(2,1 \%)\end{array}$ & 19 & $\stackrel{\overrightarrow{0}}{\hat{\sigma}}$ \\
\hline Otros adultos & $\begin{array}{c}60 \\
(14,4 \%)\end{array}$ & $\begin{array}{c}71 \\
(15,9 \%)\end{array}$ & 131 & 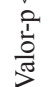 & $\begin{array}{c}9 \\
(4,7 \%)\end{array}$ & $\begin{array}{c}9 \\
(3,8 \%)\end{array}$ & 18 & $\frac{\frac{\pi}{3}}{\frac{1}{3}}$ \\
\hline $\begin{array}{l}\text { Algunos compa- } \\
\text { ñeros }\end{array}$ & $\begin{array}{c}41 \\
(9,9 \%)\end{array}$ & $\begin{array}{c}25 \\
(5,6 \%)\end{array}$ & 66 & & $\begin{array}{c}41 \\
(21,6 \%)\end{array}$ & $\begin{array}{c}29 \\
(12,2 \%)\end{array}$ & 70 & \\
\hline $\begin{array}{l}\text { Algunas compa- } \\
\text { ñeras }\end{array}$ & $\begin{array}{c}64 \\
(15,4 \%)\end{array}$ & $\begin{array}{c}32 \\
(7,2 \%)\end{array}$ & 96 & & $\begin{array}{c}22 \\
(11,6 \%)\end{array}$ & $\begin{array}{c}7 \\
(3 \%)\end{array}$ & 29 & \\
\hline No se & $\begin{array}{c}100 \\
(24,0 \%)\end{array}$ & $\begin{array}{c}188 \\
(42,2 \%)\end{array}$ & 288 & & $\begin{array}{c}53 \\
(27,9 \%)\end{array}$ & $\begin{array}{c}74 \\
(31,2 \%)\end{array}$ & 127 & \\
\hline Total & 416 & 446 & 862 & & 190 & 237 & 427 & \\
\hline
\end{tabular}

Por otra parte, en España, en los centros donde no hay estructuras, es el alumnado el que se encarga de intervenir espontánea y significativamente más que el profesorado, asegurandose que haya alguna respuesta. Paradójicamente, es en los centros españoles con estructuras donde hay más casos que se quedan sin respuesta por parte del profesorado, como si estuviera ya asignada la inmediata intervención en la ayuda.

\section{DISCUSIÓN}

La investigación contrasta las posiciones teóricas del profesorado frente al acoso y sus actuaciones concretas para pararlo percibidas por su alumnado valorando las diferencias entre contextos educativos (brasileño y español) que disponen de estructuras para el trabajo de la convivencia ética en la escuela (Equipos de Ayuda) y escenarios que no las tienen. Se divide la discusión en varios estudios.

\section{La posición del profesorado ante bullying en contextos con y sin Equipos de Ayuda}

Parece que los resultados referidos al posicionamiento moral del profesorado ante el bullying muestran una doble brecha, por una parte entre países y por otra parte entre contextos con estructuras de ayuda y sin ellas. Esta brecha puede explicarse por la marcada diferencia en la puesta en marcha de programas de convivencia ética entre ambos países (Avilés, Menin y Tabares, 2019; Menin y Avilés, 2017). Es sabida en Brasil la ausencia de políticias públicas insititucionalizadas con ese 
objetivo, lo que coincide con ese resultado. Esto ha sido respaldado por investigaciones que exploran el trabajo en valores, al denotar diferencias pedagógicas y de cultura escolar para el trabajo de los valores de forma generaliza en las escuelas, marcando trayectorias separadas y cruzadas en ambos países. Partimos de la constatación de que el trabajo en convivencia es más generalizado en las escuelas españolas que en las brasileñas, donde es testimonial. Además, en las escuelas brasileñas el modelo de formación del profesorado para el trabajo preventivo y de intervención en problemas de convivencia es muy reciente, en comparación con el modelo español.

Aquí, los datos revelan (ver Tablas 3 y 4) que el profesorado alcanza respuestas moralmente más elevadas en contextos con estructuras con Equipos de Ayuda cuando valora las dos situaciones de bullying sobre las que debe posicionarse, que cuando no existen, donde sus valoraciones se agrupan en niveles de desarrollo moral inferiores. Desde este punto de vista, la comprensión del profesorado de las formas de victimización (pasiva y provocativa) también alcanza niveles de desarrollo moral distintos (Gonçalves, 2017). Existe una mejor y mayor comprensión del profesorado de la figura de la víctima provocativa en escenarios con Equipos de Ayuda que en los que no. La propia formación y reflexión sobre la convivencia y sus dinámicas podría ayudar a los docentes a tener menos desconexiones morales ante este tipo de victimización.

Por otra parte, esto repercute en las respuestas que el profesorado y las estructuras de iguales dan frente al acoso y en el clima de aula. Cuando el tono general del trabajo en valores es explícitamente más generalizado, las diferencias de posicionamiento del profesorado frente a las situaciones de acoso son menos marcadas. Vease el caso de España (Tabla 5). Cuando la mayoría de las escuelas disponen de un trabajo intencional para la mejora de la convivencia, propiciado por la obligatoriedad de sus planes de centro, suele suceder que gran parte del profesorado incluye en sus programaciones actuaciones tendentes al logro de estos objetivos, lo que repercute de forma más directa en su forma de considerar y valorar las situciones de quiebra de la convivencia, como es el caso del bullying. Si además, ese profesorado facilita la construcción de estructuras de apoyo entre iguales entre su alumnado y recibe formación para ello, esa tarea suele repercutir en una mejora de las relaciones interpersonales (Avilés y Petta, 2018), al haber mecanismos organizados para dar respuesta a los conflictos, que el propio alumnado interioriza como plataformas útiles para resolver esos problemas, por lo que recurre a ellas siempre que lo necesita. Notese que esta tendencia puede estar detrás del dato de que es el alumnado español con estructuras de ayuda el que suele intervenir más frente al acoso. Algunos estudios lo constatan específicamente en la calidad del clima de aula (Vinha, Nunes, Togneta y Avilés, 2017) al evaluar la aplicación de programas de mejora de la convivencia ética en las escuelas en Brasil. 


\section{La actuación del profesorado ante el bullying en contextos con y sin Equipos de Ayuda}

La respuesta del profesorado frente al bullying y la calidad moral de su respuesta puede contrastarse desde dos perspectivas que no tienen por qué ser coincidentes. Lo que piensan los propios docentes cuando se les pregunta sobre el bullying y lo que hacen frente a él. La primera perspectiva se refleja en lo que contestan en el cuestionario de posicionamiento moral (un cuestionario con situaciones hipotéticas) lo que permite identificar solo los juicios de los participantes y no sus acciones propiamente dichas; y la segunda, a partir de lo que dice su alumnado cuando les ve actuar en situaciones reales, al responder algunos items del Insebull. Es sabida la discusión sobre las posibles diferencias entre lo que las personas responden como deseable frente a un dilema (situación de bullying) y lo que finalmente hacen cuando tienen que intervenir en él.

Desde esa segunda perspectiva, las respuestas del alumnado sobre el desempeño de su profesorado frente al acoso pueden ser un prisma para valorar su actuación. En el estudio se constatan diferencias significativas entre países a favor del profesorado español, y especialmente en contextos donde no hay un trabajo sobre convivencia ni estructuras de apoyo entre iguales (ver Tabla 5) donde, según el alumnado, su profesorado no actúa ante el bullying.

Por esta razón, el modelaje del profesorado interviniendo de forma explícita es tan relevante para el alumnado y para la reducción del bullying (Boulton, 1997; Garaigordobil y Oñaederra, 2010), dada la importancia de las creencias que el profesorado tiene sobre el fenómeno (Cuervo, Nenninger y Valenzuela, 2014), su nivel de autoeficacia (Souza y Tognetta, 2016) y la naturaleza de los mensajes que trasmite en el aula cuando actúa contra el acoso (Avilés, 2015). Cuando los estudiantes comienzan a percibir en su profesorado comentarios, actitudes y hechos contrarios al bullying, lo entienden como una conducta reprobable a eliminar y rechazable por parte de la insitución que representan. Especialmente son relevantes los mensajes que las y los maestros comparten con ellos cuando analizan o gestionan casos que conocen y les afectan (Saarento, Boulton y Salmivalli, 2015), escenarios en los que el acoso se suele reducir. En contextos sin este discurso, la percepción del alumnado sobre su profesorado es de inacción.

Otra dimensión crucial de la actuación del profesorado ante su alumnado es la puesta en marcha de actuaciones explícitas para resolver el acoso, tan marcadas por sus creencias sobre el fenómeno. El alumnado, especialmente el victimizado, termina creyendo en su profesorado cuando lo ve planificar y maniobrar para intervenir. En ese momento cree que puede recibir ayuda y se siente más seguro y confiado, incluso, dispuesto a hablar (Newman y Murray, 2005). Estas intervenciones y mensajes se refuerzan cuando, además, hay respaldo por parte de las familias y se acompañan de actuaciones explícitas de las estructuras de ayuda, 
con competencia social y emocional, pensadas para ello en el grupo (Avilés y Petta, 2018; Zych, Beltrán, Ortega y Llorent, 2017). De esta forma, se consiguen reunir y activar elementos efectivos para una intervención ecológica. Se trata de armonizar las fuerzas, acciones y medios que redunden de forma equilibrada -ecoconvivencia- (Avilés, 2019b) para lograr el objetivo de reducir o eliminar el acoso, rompiendo el ecosistema grupal que lo sostiene. De ahí la importancia avalada por investigaciones de intervenir con los espectadores (Bastiaensens et al., 2014) y con los roles grupales que mantienen el acoso (Salmivalli, Lagerspetz, Björkqvist, Österman y Kaukiainen, 1996).

Por otra parte, el estudio indica diferencias de respuesta en el profesorado a partir de la presencia o no de estructuras de apoyo entre iguales en sus escuelas, que pueden explicarse por la exigencia en el profesorado de un trabajo en valores para el desarrollo de estas redes, y a su grado de generalización en el sistema, mayor entre las españolas, así como por la formación que reciben los docentes para esa tarea y por el vínculo explícito que establecen, inespecífico en contextos sin estructuras explícitas, lo que acentúa las diferencias entre escuelas. Vease el caso de Brasil (Tabla 5). Esta disposición efectiva a intervenir ante situaciones de bullying para pararlo, que ocurre en mayor medida entre el profesorado español, sea que su centro disponga de estructuras de apoyo entre iguales o no, nos remite de nuevo a una brecha educativa entre países en tareas de convivencia ética y a una cultura pedagógica diferente.

Así, los datos del Insebull muestran que es en los centros españoles donde se da una participación activa del profesorado para parar el acoso más elevada que en los brasileños, donde son otras personas adultas quienes intervienen más cuando sucede. Esto estaría explicando en los docentes actitudes de desplazamiento de responsabilidad hacia otros actores (familias, técnicos especializados, otras personas que trabajan en la escuela fuera del aula), alimentadas por su abordaje aislado de los factores relevantes en el bullying, lo que rebajaría su efectividad (Pulido, Martín y Lucas, 2013). Además podría hacernos pensar en una insuficiente asunción de esa responsabilidad entre el profesorado brasileño y/o en su creencia o costumbre de derivación para su resolución.

De los datos obtenidos se desprende que es en los contextos con estructuras de ayuda donde más se implica el profesorado para parar las situaciones de acoso (significativamente más los hombres), lo que coincide con otras investigaciones (Avilés, 2006), a pesar de la feminización generalizada de la profesión (Mayer, 2015). En este sentido, la presencia o ausencia de estructuras de apoyo entre iguales como los Equipos de Ayuda marca dos características a nuestro juicio relevantes.

Por una parte, en España la presencia de estructuras entre el alumnado para prevenir el bullying reduce la percepción que tienen los estudiantes de las actuaciones de su profesorado para pararlo. En estos espacios se dan más casos sin 
respuesta. Esto podría indicar que al haber estructuras entre el alumnado para prevenir estas situaciones se podría estar relajando la atención del profesorado para estar vigilante ante las situaciones de bullying, en una especie de reparto de tareas y reducción de su intervención. Además, podría sugerir que se hace necesario reforzar por parte de los docentes, la formación y el apoyo que los Equipos de Ayuda necesitan para abordar los casos de bullying.

Por otra parte, el hecho de que en Brasil el alumnado perciba que son otros adultos y no el profesorado quienes intervienen más para parar el acoso, especialmente en espacios sin estructuras de ayuda entre iguales, nos indica la necesidad de implementar en esos espacios la generalización de la formación al profesorado y el desarrollo de programas de convivencia ética en la escuela haciendo especial incapié en el papel que juega el profesorado en la gestión de la intervención frente al bullying.

En este sentido, el área geográfica de donde se ha tomado la muestra brasileña puede ser tenida como una limitación reconocida del estudio, dado un cierto sesgo al circunscribirse solo al área de Sao Paulo y no haber representación de otras zonas de un país tan extenso como Brasil. Aun reconociendo este dato, sin embargo, hay que indicar que esta región posee cerca de veinte millones de habitantes y gran parte de estas personas viene de diferentes regiones brasileñas.

En cuanto a las prospectivas que apunta el estudio parece necesario implementar elementos de aproximación cada vez más potentes entre las concepciones del profesorado sobre el bullying y sus actuaciones específicas en el aula con su grupo de alumnado. Otras investigaciones deberían profundizar sobre si una mayor entrenamiento en técnicas específicas, más allá de una formación genérica sobre bullying, podría mejorar su capacitación para actuar más eficazmente ante el acoso. De otra parte, sería necesario también mejorar y profundizar en la formación específica y en la supervisión de las actuaciones del alumnado de los Equipos de Ayuda, mejorando asi su ajuste en la gestión de los casos de acoso y su eficacia para intervenir en ellos.

\section{CONCLUSIONES}

Se constata en ambos países que el profesorado que gestiona estructuras de ayuda entre iguales, muestra más conexiones morales frente al bullying que aquellos que no se enfrentan a la preparación, organización y formación de estas estructuras, quienes expresan más desconexiones. Estas conexiones se favorecen a partir del entrenamiento intencional, cuando se trabaja con estas estructuras para construir actuaciones antibullying en la comunidad educativa y aumentan en los docentes sus creencias de autoeficacia, tan relevantes para afrontar los casos. Al lado contrario, el profesorado que manifiesta desconexiones, suele trasmitir falta de sensibilidad ante el acoso, tender a la minimización, considerarlo un aprendizaje social, 
o incluso puede tener dificultades para identificarlo. Sin embargo, cuando el profesorado se implica y se responsabiliza en la gestión y organización de estructuras de apoyo entre iguales entre su alumnado denota un mayor grado de desarrollo moral y puede ser una manifestación de mayor preocupación ante el bullying.

Por otra parte, el alumnado percibe diferencias significativas entre países en las actuaciones de su profesorado frente al bullying en contextos donde se trabaja la convivencia ética a través de estructuras de apoyo entre iguales. Ahí, el discurso políticamente dominante se hace más presente y cotidiano. Es el modelaje comprometido en luchar contra el bullying que proyecta el profesorado el que inspira confianza y seguridad en la convivencia especialmente entre el alumnado victimizado. En ese sentido, los resultados del estudio apoyan la idea de que es en esos contextos donde se produce en los docentes un proceso de cambio y reflexión que les hace emitir respuetas ante la intimidación más efectivas y conectadas moralmente que en otros. Es precisamente en esos otros contextos donde son los iguales y otros adultos quienes asumen significativamente más esa tarea de intervención que el profesorado.

Por tanto, parece necesario fomentar y desarrollar entre el profesorado iniciativas de concienciación sobre su papel en las dinámicas del bullying, formativas que entrenen las habilides necesarias para hacerle frente con eficacia, y de gestión y de organización de estructuras de apoyo entre iguales, como los Equipos de Ayuda, que compromentan al alumnado en la responsabilidad de su propia convivencia y les hagan protagonistas moralmente comprometidos en la erradicación de esta forma de abuso, que daña la dignidad y el bienestar de la personas. Todo ello en el marco político-educativo de trabajo de la convivencia ética en la escuela desde una perspectiva de ecoconvivencia, donde se integren de forma equlibrada y complementaria las decisiones organizativas, curriculares, socioemocionales y morales que una educación integral del alumnado exige hoy.

\section{Agradecimientos / Acknowledgments:}

Los autores agradecen al profesorado y al alumnado participante en esta investigación así como a los gestores y las familias de las escuelas que aceptaron su participación en ella.

\section{Fuentes de financiamiento / Funding:}

Autofinanciado con recursos propios

\section{Rol de los autores / Authors Roles:}

JMAM: Diseño, interpretación, coordinación y redacción.

LRPT: Coordinacion, recepción y análisis de datos. 
RPD: Coordinacion, recepción y análisis de datos.

\section{Aspectos éticos / legales; Ethics / legals:}

Los autores no han incurrido en aspectos antiéticos, respetando los códigos de investigación con personas, cerciorando la protección de la confidencialidad de la información personal e institucional y asegurando el anonimato de las personas e instituciones involucradas en la muestra.

\section{Conflicto de intereses / Competing interests:}

Los autores declaran bajo juramento no haber incurrido en conflicto de interés al realizar esta investigación y artículo.

\section{REFERENCIAS}

Aceves, M.J. (2010). Seek Help from Teachers or Fight Back? Student Perceptions of Teachers' Actions during conflicts and Responses to Peer Victimization. Journal of Youth Adolescense, (39), 658-669 http://dx.doi.org/10.1007/s10964-009-9441-9

Avilés, J.M. (2006). Bullying: el maltrato entre iguales. Agresores, víctimas y testigos en la escuela. Salamanca: Amarú Ediciones.

Avilés, J.M. (2012). Manual contra el bullying. Guía para el profesorado. Lima: Ediciones. Libro Amigo.

Avilés, J.M. (2015). Proyecto Antibullying. Madrid: CEPE.

Avilés, J. M ${ }^{a}$ (2017a). Los Sistemas de Apoyo entre Iguales (SAI) y su contribución a la convivencia escolar. Innovación Educativa, 27, 5-18. doi: http://dx.doi.org/10.15304/ ie. 27.4278

Avilés, J. Ma (2019a). Los Sistemas de Apoyo entre Iguales. Barcelona: Graó.

Avilés, J. Ma (2019b). Convivir sin bullying. Madrid: Narcea.

Avilés, J. Mª y Elices, J. A. (2007). Insebull, instrumentos para la evaluación del bullying. Madrid: CEPE.

Avilés, J. M y Alonso, M ${ }^{\mathrm{a}}$ N. (2017a): Caderno de formaçao das equipes de ajuda. Alunos e alunas: Ensino Fundamental II. Americana, SP: Adonis.

Avilés, J. Ma y Alonso, Ma N. (2017b): Caderno de formaçao das equipes de ajuda. Professores e professoras: Ensino Fundamental II. Americana, SP: Adonis.

Avilés, J. Ma y Petta, R. (2018). Los Sistemas de Apoyo entre Iguales (SAI) para el fomento de la convivencia en positivo, la mejora del clima de aula y la prevención de situaciones de bullying. La experiencia de Brasil y de España. European Journal of Child Development, Education and Psychopathology, 6, (1), 5-17 https://doi.org/10.30552/ ejpad.v6i1.58 
Avilés, J. Ma, Torres, N. y Vian, M.V. (2008). Equipos de ayuda, maltrato entre iguales y convivencia escolar. Electronic Journal of Research in Educational Psychology, 6, (3), 357-376.

Avilés, J. Ma', Menin, M.S. y Tabares, M. R. (2019). Evaluación de valores sociomorales en escolares. Estudio comparativo entre alumnado español y brasileño. Revista de Educación, 384, 199-228. doi: 10.4438/1988-592X-RE-2019-384-410

Avilés, J. Ma, Cowie, H. y Alonso, Ma N. (2019). El valor de la ayuda. Equipos de Ayuda frente al bullying. Barcelona: Horsori.

Bandura, A. (1999). Moral disengagement in the perpetration of inhumanities. Personality and Social Psychology Review, 3 (3), 193-209. http://dx.doi.org/ 10.1207/s15327957pspr0303_3

Bastiaensens, S., Vandebosch, H., Poels, K, Van Cleemput, K., Desmet, A. (2014). Cyberbullying on social network sites. An experimental study into bystanders' behavioural intentions to help the victim or reinforce. Computers in Human Behavior, 31, 259271. http://dx.doi.org/10.1016/j.chb.2013.10.036

Boulton, M. J. (1997). Teachers' views on bullying definitions, attitudes and ability to cope. British Journal of Educational Psychology, 67, 223-233. http://dx.doi. org/10.1111/j.2044-8279.1997.tb01239.x

Cuervo, A., Nenninger, E. y Valenzuela, A. (2014). Creencias de docentes acerca del bullying. Perfiles Educativos, 36, (145), 51-64. https://doi.org/10.1016/S0185-2698(14)70637-3

Cowie, H. y Smith, P. (2002) . Violência nas escolas: uma perspectiva do Reino Unido. En Violência nas escolas: dez abordagens Europeias. Brasília: UNESCO. Recuperado de [http://unesdoc.unesco.org/images/0012/001287/128722por.pdf. consultado 29 de noviembre 2018].

Debarbieux, E. (2010). Lutte contre les violences et l'absentéisme scolaires. En Le Figaro. Recuperado de [www.pluslefigaro.fr/article/Debarbieux-uneespiraleinfernaledelaviolence.2010217-141717, consultado 12 de mayo de 2015].

Embrace Civility (2016). Student Survey. Embrace Civility in the Digital Age, Recuperado de [ http://embracecivility.org, consultado 12 de enero de 2017].

Gage, N., Prykanowski, D. y Larson, A. (2014). School Climate and Bullying Victimization: A Latent Class Growth Model Analysis. School Psychology Quarterly, 29, (3), 256-271. http://dx.doi.org/10.1037/spq0000064

Garigordobil, M. y Oñaederra, J.A. (2010). Los centros educativos ante el acoso escolar: actuaciones del profesorado, acciones sancionadoras y actividades de prevención. Informació Psicológica, 99, 4-18.

Gómez Rivas, F. (2011). La normativa sobre convivencia escolar en España. Revista Amazónica, 4, (1), 149-185,

Gini, G. Albiero, P, Benelli, B.y Altoé, G. (2007) Does empathy predict adolescents' bullying and defending behavior? Aggressive Behavior, 33, 467-476. http://dx.doi. org/10.1002/ab.20204 
Gonçalves, C. (2017). Engajamento e desengajamento moral de docentes em formação diante de situações de bullying envolvendo alvos típicos e provocadores. Tesis Doctoral no publicada. Joao Pessoa: UFPB.

Kohlberg, L. (1989). Estádios morales y moralización. El enfoque cognitivo-evolutivo. En Turiel, E., Enesco, I. y Linaza, J. (Comps.). El mundo social em la mente infantil (pp. 71-100) Madrid: Alianza Editorial.

Kohlberg, L. (1992). Psicologia del desarrollo moral. Bilbao: Biblioteca de Psicologia, Desclée de Brouwer.

Mayer, L. (2015). Feminización y masculinización del espacio escolar. La necesidad del "hombre" En Repositorio Digital: Universidad Politécnica Salesiana, Recuperado de [http://dspace.ups.edu.ec/handle/123456789/10990, consultado 1 mayo 2018].

Menin, M.S. y Avilés, J.Ma (2017). Adhesión a valores de convivencia en niños y adolescentes españoles y brasileños. Estudio de una investigación transcultural. En J. C. Núñez, $\mathrm{M}^{\mathrm{a}}$ C. Pérez-Fuentes, $\mathrm{M}^{\mathrm{a}}$ M. Molero, J. J. Gázquez, Á. Martos, A.B. Barragán, $\mathrm{M}^{\mathrm{a}} \mathrm{M}$. Simón (Comps.) I Congreso Internacional de Psicología, Salud y Educación. Temas actuales de investigación en las áreas de la Salud y la Educación ,52, (pp. 419-427). Oviedo: Universidad de Oviedo.

Newman, R. S. y Murray, B. J. (2005). How students and teachers view the seriousness of peer harassment: When is it appropriate to seek help? Journal of Educational Psychology, 97, 347-365. doi:10.1037/0022-0663.973.347

Olweus, D. (1998). Conductas de acoso y amenaza entre escolares. Madrid: Morata.

Piaget (1932/1994). O juizo moral na criança. São Paulo, Summus.

Pulido, R., Martín, G. y Lucas, B. (2013). Orígenes de los programas de mediación escolar: distintos enfoques que influyen en esta práctica restaurativa, Anales de Psicología, 29, (2), 385-392, Recuperado de http://dx.doi.org/10.6018/ana-lesps.29.2.132601 (consultado 26 mayo 2018).

Saarento, S., Boulton, A., \& Salmivalli, C. (2015). Reducing bullying and victimization: Student- and classroom-level mechanisms of change. Journal of Abnormal Child Psychology, 43, 61-76. doi: 10.1007/s10802-013-9841-x.

Salmivalli C, Lagerspetz M, Björkqvist K, Österman D, Kaukiainen A. (1996). Bullying as a group process. Aggress Behavior; 22: 1-15.

Salmivalli,C; Voeten, M.; Poskiparta, E.(2011). Bystanders Matter: Associations Between Reinforcing, Defending, and the Frequency of Bullying Behavior. En Journal of Clinical Child \& Adolescent Psychology, 40 (5), 668-676. http://dx.doi.org/10.1080 /15374416.2011.597090

Sela-Shayovitz, R. (2009). Dealing with school violence: The effect of school violence prevention training on teachers' perceived self-efficacy in dealing with violent events. Teaching and Teacher Education, 25 (8), 1061-1066. http://dx.doi.org/10.1016/j. tate.2009.04.010

Souza, R. y Tognetta, L. (2016). As creencias de auto-eficacia de espectadores en las situaciones de bullying: ¿Porque Ayudar? $\mathrm{M}^{\mathrm{a}} \mathrm{C}$. Pérez-Fuentes, J. J. Gázquez, $\mathrm{M}^{\mathrm{a}}$ 
M. Molero, Á. Martos, Ma M. Simón y A. B. Barragán (Comps.) La Convivencia Escolar: Un acercamiento multidisciplinar, II, (pp. 61-66). Almería: Universidad de Almería.

Tognetta, L.R.P., Avilés, J.M., Rosario, P. y Alonso, N. (2015). Desengajamentos morais, autoeficácia e bullying: a trama da convivência. Estudios e investigación em psicologia y educación, 2 (1), 30-34. doi: 10.17979/reipe.2015.2.1.714.

Tognetta, L., Avilés, J.Ma, Gonçalves, C., Andrade, F., Daud, R. y Santos, N. (2019). Validação de instrumento sobre Engajamento e Desengajamento Moral de docentes diante do bullying na escola. Artículo entregado para publicación.

Vinha, T., Nunes, C., Tognetta, L. y Avilés, J.Mª (2017). Um programa visando a convivencia ética e a melhoria do clima escolar realizado em escolas brasileiras. En J. C. Núñez, $M^{a}$ C. Pérez-Fuentes, M M. Molero, J. J. Gázquez, Á. Martos, A.B. Barragán, M M. Simón (Comps.) I Congreso Internacional de Psicología, Salud y Educación. Temas actuales de investigación en las áreas de la Salud y la Educación, 17, (pp. 199-228) Oviedo: Universidad de Oviedo.

Zaitegi, N., Fernandez, I., Uruñuela, P., Avilés, J.M., Boqué, M.C., y Gómez, F. (2010). La educación en y para la convivencia positiva en España. Revista iberoamericana sobre calidad, eficacia y cambio en la educación, 8, (2), 93-132.

Zych, M., Beltrán, M., Ortega, R. y Llorent, V. (2018). Competencias sociales y emocionales de adolescentes involucrados en diferentes roles de bullying y cyberbullying. En Revista de Psicodidáctica, Recuperado de [http://www.sciencedirect.com/science/ article/pii/ S1136103417301429 doi: https://doi.org/10.1016/j.psicod.2017.12.001. 
